\title{
Creation of stable Pseudomonas aeruginosa promoter-reporter fusion mutants using linear plasmid DNA transformation
}

Ping Chen and Kai P. Leung*

\begin{abstract}
Background: Pseudomonas aeruginosa is an important opportunistic human pathogen that is commonly encountered clinically in different types of infections. Reporter-gene systems and construction of mutants defective in specific functions are useful tools for studying the cellular physiology and virulence of this organism. The common mutant construction process requires constructing target alleles into large size suicide vector(s) for transformations, and extra steps involved in resolving merodiploids. Here we describe a new approach using linearized plasmid transformation for creating a green fluorescent protein (GFP) reporter gene system to study promoter activities in $P$. aeruginosa.

Findings: We successfully created promoter-reporter fusion plasmids for studying the promoter activity of virulence genes in $P$. aeruginosa. The promoter of exoenzyme $S$ (a virulence factor) was used in preparation of these fusion plasmids. These fusion plasmids were linearized and used directly to transform P. aeruginosa. Stable P. aeruginosa chromosomally integrated promoter-reporter fusion mutants were obtained. We demonstrated that the promoter of Exoenzyme $S$ gene was activated when P. aeruginosa was grown in a biofilm state, as evidenced by the expression of GFP in these biofilm cells.
\end{abstract}

Conclusion: Direct transformation with linearized plasmid DNA provides another avenue to create P. aeruginosa mutants. This new approach eliminates the use of suicide vector(s) for creating $P$. aeruginosa mutants, and thus speeds up the process mutant construction.

Keywords: Pseudomonas aeruginosa, Promoter activity, Green fluorescent protein, Linear DNA transformation

\section{Background}

The ubiquitous Gram-negative Pseudomonas aeruginosa is an important opportunistic human pathogen. This organism has a large genome of 6.3 million nucleotides [1], and causes both life-threatening acute infections as shown in burn patients, and chronic lung infections as in cystic fibrosis patients [2]. P. aeruginosa lives in two different life styles, planktonic and biofilm, which are thought to be associated with acute infections and chronic infections, respectively. The expression of virulence in $P$. aeruginosa, which can be influenced by

\footnotetext{
${ }^{*}$ Correspondence: kai.p.leung.civ@mail.mil
}

Dental and Craniofacial Trauma Research and Tissue Regeneration, US Army Institute of Surgical Research, JB Fort Sam Houston, 3650 Chambers Pass, Bldg 3610, San Antonio, TX 78234-6315, USA environmental signals, is tightly controlled by complex regulatory networks. About $8.4 \%$ of predicted P. aeruginosa genes are involved in regulation, which is among the highest proportion of predicted regulatory genes observed in sequenced bacterial genomes [1]. Therefore, it is important to understand the regulatory pathways controlling the expression of virulence in $P$. aeruginosa under different conditions and time. This can be accomplished by studying the promoter activities of virulence genes at both individual and global gene expression levels (such as RNA sequencing).

To study the promoter activity of a particular gene, the promoter is commonly fused to a reporter gene [such as green fluorescent protein (GFP) gene] in a replicative plasmid in order to obtain the highest levels of 
fluorescence. The resultant plasmid is introduced into the host bacterium, and the activities of the reporter gene are measured under the desired test conditions. To prevent loss of the plasmid, antibiotic is added into the growth medium to maintain the selection pressure. However, in some circumstances (such as polymicrobial interaction studies), the continuous presence of antibiotics to maintain the selection pressure is not desirable because some interacting strains could be susceptible to the antibiotic. In addition, antibiotics themselves can have untoward effect on gene expression circuits. Therefore, it is more preferable to have the fused promoter-reporter element stably integrated into the bacterial chromosome.

Creating stable chromosomal insertion/deletion mutants involves several steps [3,4]. Firstly, target alleles that are often tagged with an antibiotic resistance gene are inserted into a suicide plasmid. Secondly, the resultant suicide plasmid is delivered into the host by electroporation or conjugation, followed by recombination of the plasmid-borne target into the chromosome. Finally, correct mutants are obtained by resolving the merodiploids that result from chromosomal integration of the suicide plasmid by a single crossover. This latter procedure uses a counter-selectable marker such as Bacillus subtilis $s a c B$ (sucrose counter selection). Some drawbacks of this conventional approach include: (1) difficulties encountered in $s a c B$ counter-selection due to issues of stringency; and (2) cloning difficulties due to the relatively large sizes of suicide plasmids themselves and few available cloning sites. In this report, we showed that $P$. aeruginosa mutants can be directly created by electroporation of high concentrations of linearized cloning plasmid DNA, eliminating the use of a suicide plasmid.

\section{Methods}

\section{Growth conditions for bacterial strains and plasmids manipulations}

Escherichia coli $5 \alpha$ strains used for subcloning and plasmid isolation were grown in Luria-Bertani (LB, $10 \mathrm{~g} / \mathrm{l}$ of tryptone, $10 \mathrm{~g} / \mathrm{l}$ of $\mathrm{NaCl}$ and $5 \mathrm{~g} / \mathrm{l}$ of yeast extract) medium at $37^{\circ} \mathrm{C}$ in the presence of the appropriate selective substance $(10 \mu \mathrm{g} / \mathrm{ml}$ of Gentamicin), or in low salt LB medium ( $10 \mathrm{~g} / \mathrm{l}$ of tryptone, $5 \mathrm{~g} / \mathrm{l}$ of $\mathrm{NaCl}$ and $5 \mathrm{~g} / \mathrm{l}$ of yeast extract) containing antibiotic zeocin $(50 \mu \mathrm{g} / \mathrm{ml})$ (Invitrogen, Carlsbad, CA). E. coli plasmid DNA isolations were carried out by the QIAprep Spin Miniprep Kit (QIAGEN, Valencia, CA). Routine procedures were employed for manipulation of DNA. Unless specified, $P$. aeruginosa $\mathrm{PAO} 1$ was grown in $\mathrm{LB}$ and clinical strains were grown in brain heart infusion (BHI).

\section{Promoter-reporter fusion plasmid constructions}

Steps for inserting the fused promoter-reporter element into the $P$. aeruginosa chromosome are illustrated in Fig. 1. Briefly, an approximate $2.2 \mathrm{~kb}$ intergenic region between open reading frames (ORFs) of PA3835 and PA3836-a region without any transcription based on our unpublished RNA Sequencing (RNA-Seq) data-was PCR-amplified using primers (listed in Additional file 1: Table S1) containing XhoI sites, and cloned into cloning vector $\mathrm{pDONR/Zeo} \mathrm{from} \mathrm{Invitrogen.} \mathrm{Using} \mathrm{the} \mathrm{site-}$ directed mutagenesis method, two unique sites, BglII and SpeI, were created sequentially with BglII site located in the middle of the intergenic region, and SpeI site located at $10 \mathrm{bp}$ up-stream of the BglII site. Using plasmid pJQ200 (ATCC ${ }^{\circledR} 77482$ ) as a template, the gentamicinresistance gene with its own promoter, was PCR amplified with $B a m H I$ site-containing primers, digested with $B a m H I$, and inserted at the BglII site in the intergenic

A $2.2 \mathrm{~kb}$ intergenic region between

ORFs PA3835 and PA3836

a
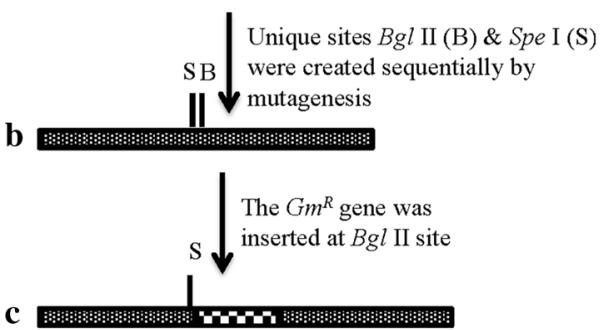

$\mathrm{Gm}^{R}$

Promoterless NT- $g f p$ was

inserted at Spe I site

d

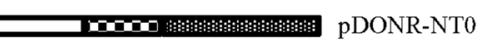

$g f p \quad G m^{R}$

Promoter element (P) of

PA3841 was inserted up-

stream of $g f p$

e

$p \quad g f p \quad G m^{R}$

Fig. 1 Schematic of inserting the fused promoter-reporter element into $P$. aeruginosa. a A roughly $2.2 \mathrm{~kb}$ intergenic region between ORFs of PA3835 and PA3836 was PCR amplified and cloned into vector pDONR/Zeo obtained from Invitrogen-Thermo Fisher Scientific (Grand Island, NY). b Two unique sites, Bg/ll (B) and Spe I (S) were sequentially created by site-directed mutagenesis with the Bg/ll site located in the middle of the intergenic region, and the Spel site located at $10 \mathrm{bp}$ upstream of the Bg/ll site. c The gentamicin resistance gene $\left(\mathrm{Gm}^{R}\right)$ was amplified by PCR and inserted at the Bg/ll site. d The long half-life version of $g f p$ was inserted at the Spel site, generating plasmid pDONR-NTO. e Promoter of PA3841was inserted upstream of gfp in pDONR-NTO, creating construct pDONR-NT3841P. The final plasmid was digested with Xhol, dephosphorylated, column purified and used to electroporate wild-type P. aeruginosa PAO1 according to a published method [6]. The promoter-reporter element was inserted into the chromosome by a double cross-over recombination event. Primers used were listed in Additional file 1: Table S1 
region. A long half-life version of $g f p$ containing its own ribosome binding site with surrounding $r r n B$ T1 terminator sequences was amplified by PCR from vector pPROBE-NT [5], and inserted at the SpeI site upstream of the gentamicin-resistance marker. This construct was designated as pDONR-NT0. It has three unique restriction enzyme cutting sites (XbaI, KpnI \& EcoRI) for cloning of any promoter fragment to be studied into the upstream of the reporter $g f p$.

The sequence of the test promoter was PCR amplified and inserted into upstream of the reporter $g f p$ at the unique site(s) $(X b a \mathrm{I}, K p n \mathrm{I} \& E c o R \mathrm{I})$. The resultant plasmid was digested with $X h o I$ and dephosphorylated using shrimp alkaline phosphatase (rSAP) to prevent selfreligation. The treated and linearized DNA was column purified using Wizard SV PCR clean-up mini-column from Promega (Madison, WI), and used directly for electroporation.

\section{$P$. aeruginosa transformation}

$P$. aeruginosa electro-competent cells were prepared according to a previously published method [6]. Cells from $6 \mathrm{ml}$ of an overnight culture grown in LB or BHI broth with an optical density of approximately 1.3 $(\lambda=600 \mathrm{~nm})$ were harvested, washed, and re-suspended in $100 \mu \mathrm{l}$ room temperature $300 \mathrm{mM}$ sucrose. The cell suspension was mixed with approximately $5 \mu \mathrm{g}$ Wizard SV PCR clean-up mini-column-purified linearized DNA. Transformation was carried out using an ECM630 Electro Cell Manipulator from BTX (Holliston, MA) in a $2 \mathrm{~mm}$ gap width electroporation cuvette with the following settings: $25 \mu \mathrm{F}, 189 \Omega$, and $2.5 \mathrm{kV}$. Following the immediate addition of Super Optimal Broth (SOC) medium $(0.6 \mathrm{ml})$ after electroporation, cells were transferred to a $17 \times 100 \mathrm{~mm}$ style $14 \mathrm{ml}$ polypropylene round-bottom tube, and incubated for $2 \mathrm{~h}$ at $37^{\circ} \mathrm{C}$ with shaking at $150 \mathrm{rpm}$. The entire mixture was plated on four PIA (Pseudomonas isolation agar; Becton, Dickinson and Co.) plates, each containing $40 \mu \mathrm{g} / \mathrm{ml}$ gentamicin. After overnight incubation, colonies were analyzed for proper double crossover recombination by colony PCR based on the sizes of the PCR products.

\section{Growing $P$. aeruginosa biofilms in the bioflux system}

The mid-log phase $P$. aeruginosa culture was passed through a $5-\mu \mathrm{m}$ syringe filter (Pall Corporation, Ann Arbor, MI) to reduce the number of aggregates. For inoculation, the culture was adjusted to an optical density of 0.1 at the wavelength of $600 \mathrm{~nm}\left(\mathrm{OD}_{600}\right)$. The channels of a 48-well microplate from the Bioflux system (Fluxion Biosciences, South San Francisco, CA) were primed with media, followed by inoculation of each channel with $50 \mu \mathrm{l} P$. aeruginosa suspension $\left(\mathrm{OD} 600_{\mathrm{nm}}=0.1\right)$. After
$2 \mathrm{~h}$ of attachment, the channels were perfused with $50 \%$ BHI medium at a shear flow of $0.55 \mathrm{dyn} / \mathrm{cm}^{2}$ to initiate the growth of biofilms. Biofilm formation and green fluorescence were monitored in real time using the LSM710 confocal microscope (Carl Zeiss MicroImaging, Thornwood, NY).

\section{Results and discussion}

To study the promoter activities, we constructed a promoter-reporter fusion plasmid pDONR-NT0 (see Fig. 1). This plasmid contains three unique restriction enzyme cutting sites (XbaI, KpnI \& EcoRI) for cloning of any promoter fragment to be studied into the upstream of the long half-life version $g f p$ reporter. Gentamicin-resistance marker was used for selection. The insertion site was chosen to be located at the intergenic region between open reading frames of PA3835 and PA3836-a region without any transcription based on our unpublished RNA Sequencing (RNA-Seq) data. Basic local alignment search tool (BLAST) results showed that although the sequence could vary, most, if not all, $P$. aeruginosa strains have this intergenic region. Because this region is non-coding and lack of transcription activity; therefore, this insertion site is not likely to interfere with other gene functions.

To prove the concept of transformation using linearized plasmid DNA, we used the promoter element of Exoenzyme $\mathrm{S}$ structural gene, a type III secretion effector and an important virulence factor for $P$. aeruginosa [7]. We amplified this promoter by PCR and cloned it into pDONR-NT0 (see Fig. 1) to create pDONR-NT3841P. We transformed $5 \mu \mathrm{g}$ of each of these linearized plasmids into $P$. aeruginosa PAO1 competent cells. The rational for using $5 \mu \mathrm{g}$ total DNA is that this amount has consistently yielded enough transformants for downstream characterization in our preliminary determinations. In addition, it has been shown that the number of recombinants generated from $5 \mu \mathrm{g}$ total DNA is close to the maximum number of transformants that can be obtained in other Gram-negative bacteria [8]. Eight and twelve transformants were obtained from the pDONR-NT0 and pDONR-NT3841P transformation respectively. PCR analyses confirmed that about $50 \%$ of the transformants were correct double crossover recombinants (Table 1). The recombinants were designated as $P$. aeruginosa NT0 and NT3841P respectively. There was no difference in growth patterns between the double crossover recombinants obtained $v s$. wild-type $P$. aeruginosa PAO1 (data not shown). The incorrect double crossover recombinants were spontaneous gentamicin-resistant mutants and single crossover recombinants that were likely resulted from the incomplete enzyme digestions.

To demonstrate the effectiveness of the promoterreporter fusion system created, we compared the 
Table 1 Recombination frequencies of $P$. aeruginosa PAO1 transformation using linearized plasmid DNA

\begin{tabular}{|c|c|c|c|}
\hline Constructs $^{a}$ & $\begin{array}{l}\text { \# Of transfor- } \\
\text { mants }\end{array}$ & $\begin{array}{l}\text { \# Of correct } \\
\text { recombinants }\end{array}$ & $\begin{array}{l}\text { Correct ratio } \\
(\%)^{b}\end{array}$ \\
\hline pDONR-NTO & 8 & 4 & 50 \\
\hline $\begin{array}{l}\text { pDONR- } \\
\text { NT3841P }\end{array}$ & 12 & 6 & 50 \\
\hline pDONR-NT45P & 7 & 3 & 42.8 \\
\hline $\begin{array}{l}\text { pDONR- } \\
\text { NT3194P }\end{array}$ & 10 & 5 & 50 \\
\hline pDONR-ASVO & 7 & 3 & 42.8 \\
\hline $\begin{array}{l}\text { pDONR- } \\
\text { ASV3841P }\end{array}$ & 10 & 4 & 40 \\
\hline pDONR-ASV45P & 11 & 4 & 36.4 \\
\hline $\begin{array}{l}\text { pDONR- } \\
\text { ASV3194P }\end{array}$ & 12 & 6 & 50 \\
\hline $\begin{array}{l}\text { pDONR- } \\
\text { ASV4228P }\end{array}$ & 7 & 3 & 42.8 \\
\hline
\end{tabular}

a NT long half-life version of GFP. ASV short half-life (110 min) version of GFP. 0 no promoter element. $P$ promoter element from that particular ORF (e.g. 3841P-promoter from ORF PA3841)

b Ratio \# of correct recombinants per \# of transformants

expression of green fluorescent protein between recombinant strains NT0 (promoterless control) and NT3841P which contained the promoter of exoenzyme $S$ gene. Our transcriptome profiling (RNAseq) showed that the promoter of exoenzyme $\mathrm{S}$ gene was activated when $P$. aeruginosa grew in a biofilm. Indeed, as compared to the promoterless control, we observed increased fluorescence from the NT3841P recombinant grown as biofilms in the Bioflux System (Fig. 2). Although biofilm formation and type III secretion have been shown to be reciprocally regulated in some publications [9], biofilms and type III secretion are not mutually exclusive in Pseudomonas aeruginosa. Under some growth condition(s), the type III effector proteins (such as ExoS and ExoT) were expressed in biofilm cells, but not in the planktonic cells [10]. The discrepancy may be caused by different growth conditions used in the studies. The Bioflux system that we used to grow biofilm is a continuous-flow system which more closely mimics the in vivo conditions. Our results are in agreement with that of Mikkelsen et al., showing increased ExoS expression in biofilm cells grown in a continuous-flow system [10].

To further confirm the effectiveness of the linearized plasmid DNA transformation approach, we transformed P. aeruginosa $\mathrm{PAO} 1$ with other constructs containing the promoter of a hypothetical protein (PA45) gene, the promoter of a phosphogluconate dehydratase (PA3194) gene, and the promoter of pyochelin (PA4228) gene using different versions of $g f p$ reporter genes. For each transformation, although there are variations in transformation efficiencies, we were able to obtain correct double crossover recombinants of these different constructs (Table 1). However, we were not able to monitor the activities of these promoters using our constructed system, possibly due to the weakness of the promoters and/or the test conditions are not optimal.

To determine if we can create deletion mutant using this linearized plasmid DNA transformation approach, a small none-coding regulatory RNA deletion plasmid was constructed (by Dr. Rajasekh Karna and Dr. Christine Miller in our laboratories) on the pCR2.1 vector backbone. The plasmid was linearized and transformed into P. aeruginosa PAO1 competent cells. Two hundred and eight gentamicin-resistant transformants were obtained. PCR analyses of randomly picked eight transformants showed that all were correct double crossover deletion mutants. We also used this linearized plasmid to transform a highly virulent clinical strain $P$. aeruginosa strain 12-4-4-59 [11]. We obtained eight correct double crossover deletion mutants. This result suggests that this linearized plasmid DNA transformation approach is not limited to PAO1 strain only.

Recombination efficiency could be influenced by the size of both the flanking region and the inserted nonhomologous element, and by the surrounding sequence contexts. As the size of the inserted non-homologous DNA increases, the efficiency decreases exponentially; and once it reaches about $6 \mathrm{~kb}$, the recombination efficiency is not detectable [12]. In contrast, as the flanking region size increases, the recombination efficiency increases. However, once the flanking region size reaches $1 \mathrm{~kb}$, the recombination efficiency is not further improved [12]. In our case, the sizes of both flanking regions are $\sim 1 \mathrm{~kb}$, whereas the inserted non-homologous DNA is $\sim 3 \mathrm{~kb}$. Therefore, the low recombination efficiency observed is not likely due to the length of the flanking regions, but more likely due to the large size of the inserted element. The fact that we obtained more deletion mutants supports this. We were not able to improve the transformation efficiency by growing $P$. aeruginosa at $42{ }^{\circ} \mathrm{C}$ either for overnight or just for $2 \mathrm{~h}$ before performing the transformation. The presence of the vector in the total electroporated DNA preparation should not contribute to the observed low recombination efficiency either. In contrary, it may enhance recombination rates by saturating endogenous nucleases. It has been shown that the presence of carrier oligonucleotides increased the frequencies of recombination in both Gram-negative bacteria [8] and yeast [13].

Our results showed that $P$. aeruginosa mutants could be generated by electroporation of high concentrations of linearized cloning plasmid DNA. It has been reported that $P$. aeruginosa mutants could be created 

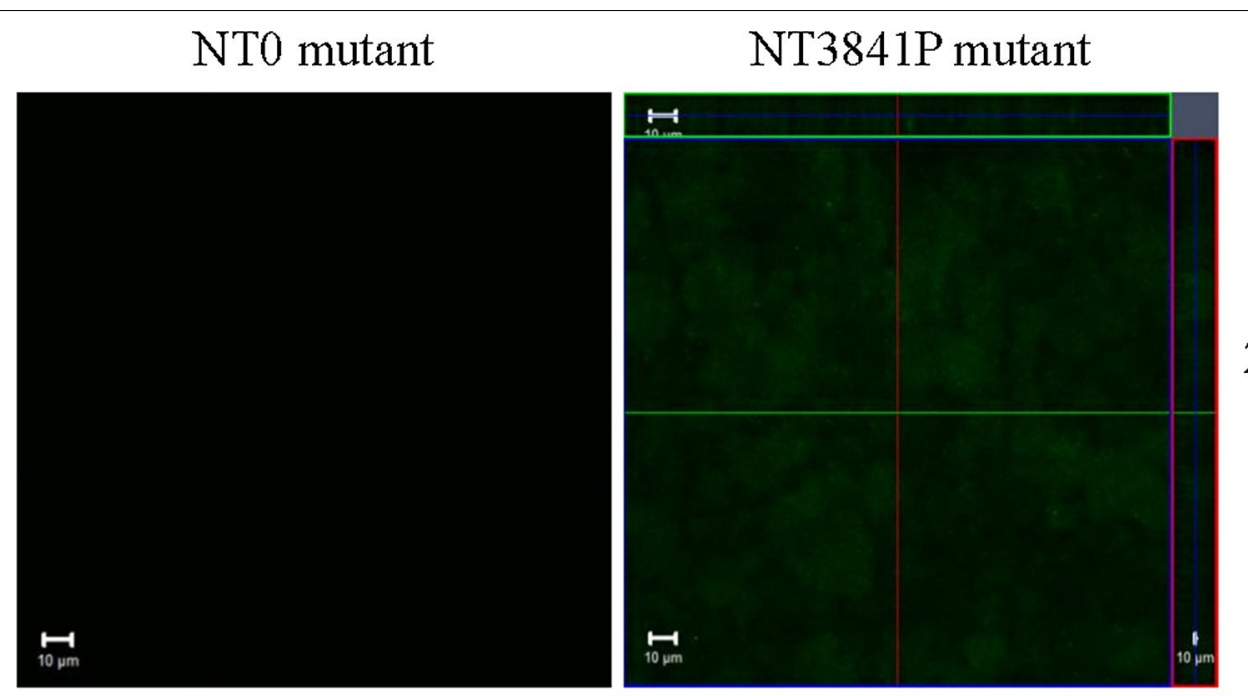

\section{$25 \mathrm{hr}$}
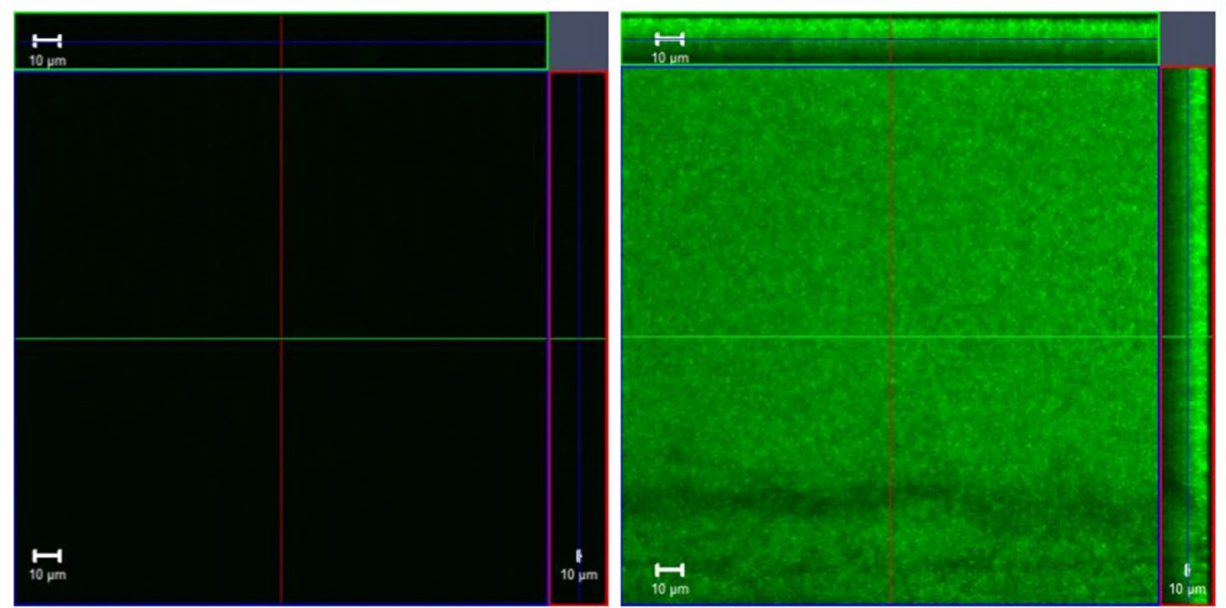

$45 \mathrm{hr}$

Fig. 2 The promoter of Exoenzyme $S$ gene (PA3841) was activated when P. aeruginosa cells were in the biofilm state. Recombinants P. aeruginosa NT0 \& NT3841P were grown at $37^{\circ} \mathrm{C}$ in a 48-well microplate Bioflux system (Fluxion Biosciences, South San Francisco, CA) with a shear flow of $0.55 \mathrm{dyn} / \mathrm{cm}^{2}$ using $50 \% \mathrm{BHI}$ (Brain Heart Infusion) medium. Images were captured after 25- and 45-h growth using a confocal microscope (LSM710; Carl Zeiss Microlmaging, Thornwood, NY) with the excitation wavelength set at 488-nm. Left panels and right panels are representative fluorescence images of NT0 and NT3841P mutants respectively. The expression of GFP was increased in the biofilms formed by the NT3841P recombinant, but not in the biofilms formed by the control NTO recombinant. Bars represent $10 \mu \mathrm{m}$

by electroporation of high concentrations of linear DNA, such as chromosomal DNA [6], PCR fragments [14], and single stranded synthetic oligonucleotides [15]. Compared to other forms of linear DNA, this linear plasmid DNA approach has two advantages: Firstly, this approach is not limited to any particular vector. Thus, it would be more convenient to manipulate a target region due to wider selections of vectors. Secondly, because cloning vectors are usually medium-tohigh copy number plasmids, it is easier to obtain large quantities of plasmid DNA needed for the transformations. Compared to the conventional mutant generating approach that requires the use of suicide vector(s), this linear plasmid DNA approach eliminates the difficult subcloning steps into the suicide vector and the time consuming steps of resolving merodiploids resulted from single crossover events. One limitation of using linear plasmid DNA is the low transformation efficiencies. However, the number of correct recombinants obtained is still more than needed for downstream characterization.

\section{Conclusion}

Direct transformation with linearized plasmid DNA provides another avenue to create $P$. aeruginosa mutants. This new approach eliminates the use of suicide vector(s) for creating $P$. aeruginosa mutants, and thus facilitates the study of this opportunistic pathogen. 


\section{Additional file}

Additional file 1: Table S1. Primers used in this study.

\section{Authors' contributions}

PC designed and performed the experiments. PC and KPL interpreted the data. PC and KPL wrote the manuscript. All authors read and approved the final manuscript.

\section{Acknowledgements}

This work was supported by the US Army Medical Research and Materiel Command, Combat Casualty Care Research Directorate.

\section{Competing interests}

The authors declare that they have no competing interests.

\section{DOD disclaimer}

The opinions or assertions contained herein are the private views of the authors and are not to be construed as official or as reflecting the views of the Department of the Army or the Department of Defense.

Received: 21 November 2015 Accepted: 17 June 2016

Published online: 24 June 2016

\section{References}

1. Stover CK, Pham XQ, Erwin AL, Mizoguchi SD, Warrener P, Hickey MJ, et al. Complete genome sequence of Pseudomonas aeruginosa PAO1, an opportunistic pathogen. Nature. 2000;406(6799):959-64.

2. Bodey GP, Bolivar R, Fainstein V, Jadeja L. Infections caused by Pseudomonas aeruginosa. Rev Infect Dis. 1983;5(2):279-313.

3. Filloux A, Ramos JL. Pseudomonas methods and protocols. New York: Humana Press; 2014.
4. Muhl D, Filloux A. Site-directed mutagenesis and gene deletion using reverse genetics. Methods Mol Biol. 2014;1149:521-39.

5. Miller WG, Leveau JH, Lindow SE. Improved gfp and inaZ broadhost-range promoter-probe vectors. Mol Plant Microbe Interact. 2000;13(11):1243-50.

6. Choi KH, Kumar A, Schweizer HP. A 10-min method for preparation of highly electrocompetent Pseudomonas aeruginosa cells: application for DNA fragment transfer between chromosomes and plasmid transformation. J Microbiol Methods. 2006;64(3):391-7.

7. Yahr TL, Hovey AK, Kulich SM, Frank DW. Transcriptional analysis of the Pseudomonas aeruginosa exoenzyme $S$ structural gene. J Bacteriol. 1995;177(5):1169-78.

8. Swingle B, Markel E, Costantino N, Bubunenko MG, Cartinhour S, Court DL. Oligonucleotide recombination in Gram-negative bacteria. Mol Microbiol. 2010;75(1):138-48.

9. Goodman AL, Kulasekara B, Rietsch A, Boyd D, Smith RS, Lory S. A signaling network reciprocally regulates genes associated with acute infection and chronic persistence in Pseudomonas aeruginosa. Dev Cell. 2004;7(5):745-54

10. Mikkelsen H, Bond NJ, Skindersoe ME, Givskov M, Lilley KS, Welch M. Biofilms and type III secretion are not mutually exclusive in Pseudomonas aeruginosa. Microbiology. 2009;155(Pt 3):687-98.

11. Walker HL, Mason AD Jr, Raulston GL. Surface infection with Pseudomonas aeruginosa. Ann Surg. 1964;160:297-305.

12. Kung SH, Retchless AC, Kwan JY, Almeida RP. Effects of DNA size on transformation and recombination efficiencies in Xylella fastidiosa. Appl Environ Microbiol. 2013;79(5):1712-7.

13. Yamamoto T, Moerschell RP, Wakem LP, Ferguson D, Sherman F. Parameters affecting the frequencies of transformation and co-transformation with synthetic oligonucleotides in yeast. Yeast. 1992;8(11):935-48.

14. Lesic B, Rahme LG. Use of the lambda red recombinase system to rapidly generate mutants in Pseudomonas aeruginosa. BMC Mol Biol. 2008;9:20.

15. Agnello $M$, Wong-Beringer $A$. The use of oligonucleotide recombination to generate isogenic mutants of clinical isolates of Pseudomonas aeruginosa. J Microbiol Methods. 2014;98:23-5.

\section{Submit your next manuscript to BioMed Central and we will help you at every step:}

- We accept pre-submission inquiries

- Our selector tool helps you to find the most relevant journal

- We provide round the clock customer support

- Convenient online submission

- Thorough peer review

- Inclusion in PubMed and all major indexing services

- Maximum visibility for your research

Submit your manuscript at www.biomedcentral.com/submit 